\title{
Ionisation et émission $X$ d'un jet puisé d'Argon irradié par des impulsions laser femtosecondes
}

\author{
C. Stenz, F. Blasco, R. Brückner, F. Amiranoff*, P. Audebert*, E. De Wispelaere*, \\ J.P. Geindre*, J.C. Gauthier*, V. Malka*, A. Dos Santos**, G. Rey**, A. Mysyrowicz** \\ et A. Antonetti**
}

Groupe de Recherche sur l'Energétique des Milieux Ionisés, Associé au CNRS, Université d'Orléans, 45067 Orléans, France

* Laboratoire d'Utilisation des Lasers Intenses, Associé au CNRS, Ecole Polytechnique, 91128 Palaiseau, France

** Laboratoire d'Optique Appliquée ^Associé au CNRS, Ecole Nationale Supérieure des Techniques Avancées, 91120 Palaiseau, France

\begin{abstract}
On présente une étude de l'ionisation optique de l'Argon à une pression voisine de l'atmosphère, par une impulsion laser femtoseconde avec un éclairement de 2-3 $10^{\wedge} \mathrm{W} / \mathrm{cm}^{2}$. L'effet de défocalisation, observé dans l'Argon statique à une pression de 1 bar, est notablement réduit par la mise en oeuvre d'un jet puisé, d'une densité de 1-3 $10^{19}$ atomes/cirA L' émission Ka et le taux d'ionisation de l'Argon présentent un maximum très localisé pour une focalisation de l'impulsion laser en bord de jet. L'état d'ionisation atteint dans ces conditions par l'Argon est de 5 à 6 , en bon accord avec les prédictions d'un modèle d'ionisation par effet tunnel pour un éclairement laser de $1 \mathrm{O}^{\wedge} \mathrm{W} / \mathrm{cm}^{2}$. L'émission Ka est interprétée comme résultant de l'ionisation des couches internes de l'Argon par des électrons dont l'énergie est acquise par le mouvement d'oscillation dans le champ laser intense.
\end{abstract}

\section{INTRODUCTION}

La propagation d'une impulsion laser ultra-courte et intense dans un gaz à une pression de 1' atmosphère est modifiée par l'ionisation optique induite par le champ laser $(1,2)$. L'effet combiné de l'ionisation et de la réfraction contribue à limiter l'éclairement laser et la densité électronique maximum atteinte dans la zone focale (zone de Rayleigh). La mise en oeuvre de jets haute pression peut permettre d'atteindre au foyer des éclairements proches de ceux atteints dans le vide.

\section{DESCRIPTION DE L'EXPERIENCE}

On utilise un laser à colorants qui délivre, à une fréquence de $10 \mathrm{~Hz}$, une impulsion de $80 \mathrm{fs}$ de durée, d'énergie comprise entre 1 et $3 \mathrm{~mJ}$ et de $620 \mathrm{~nm}$ de longueur d'onde. L'impulsion laser est focalisée dans le gaz au moyen d'un lentille de $80 \mathrm{~mm}$ de focale ouverte à $\mathrm{f} / 3$. Le flux laser maximum dans le vide est de $2-3 \hat{\mathrm{I}}^{1} \wedge \mathrm{W} / \mathrm{cm}^{2}$. Les jets sont des bouffées d' Argon d'une durée de $5 \mathrm{~ms}$ produites par ouverture, au moyen d'une électro-vanne rapide, d'une chambre haute pression (80-100 bars). L'écoulement du gaz s'effectue dans une buse de Laval conçue pour produire des jets supersoniques (Mach 4 à 5) de faible divergence. Le diamètre du jet est de $4 \mathrm{~mm}$. L'impulsion laser et l'ouverture de l'électro-vanne sont synchronisés par une horloge à $10 \mathrm{~Hz}$. Les diagnostics optiques utilisent une sonde laser de $70 \mathrm{fs}$ de durée et de $570 \mathrm{~nm}$ de longueur d'onde. Le profil de densité d'Argon dans le jet est obtenu par interférométrie Mach-Zehnder. L'imagerie ombroscopique du plasma d'Argon permet de caractériser la focalisation du faisceau laser dans la zone focale (3). L'étude du 
spectre laser transmis, présente un décalage vers le bleu dû à la variation rapide de la densité électronique qui résulte de la dynamique de l'ionisation laser ( Fig 1). L'état d'ionisation de l'Argon est déduit de la mesure de densité électronique par interférométrie Moiré avec une résolution spatiale de 5 microns et une résolution temporelle de $70 \mathrm{fs}$. L' émission $X$ est observée au moyen d'une CCD refroidie fonctionnant en mode comptage de photons avec une résolution de $50 \mathrm{eV}(4)$.

\section{PRINCIPAUX RESULTATS}

La forte défocalisation du faisceau laser observée dans l'Argon à une pression de 1 bar (3) contribue à réduire l'intensité laser dans la zone de Rayleigh. L'état d'ionisation de l'Argon mesuré dans ces conditions est de 1 à 2 et correspond à celui que l'on peut prévoir pour un éclairement laser sensiblement 10 fois plus faible que celui mesuré sous vide. Des résultats similaires sont obtenus pour une focalisation au centre du jet.

L'effet de réfraction est par contre notablement réduit pour une focalisation en bord de jet. L'on atteint dans ce cas un état d'ionisation de 5 à 6 , comparable à celui que l'on peut prévoir théoriquement pour une intensité laser de $1016 \mathrm{~W} / \mathrm{cm}^{2}$ (Fig 1).

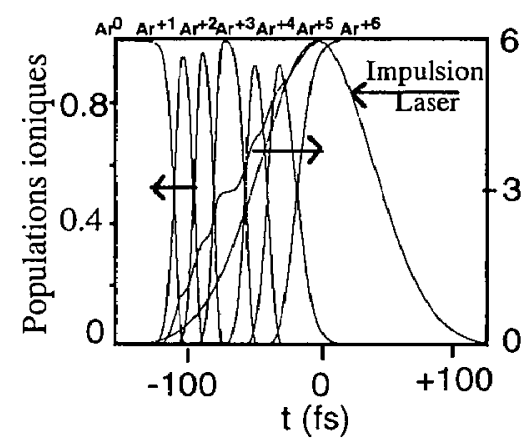

Fig 1. Ionisation tunnel de l'Argon

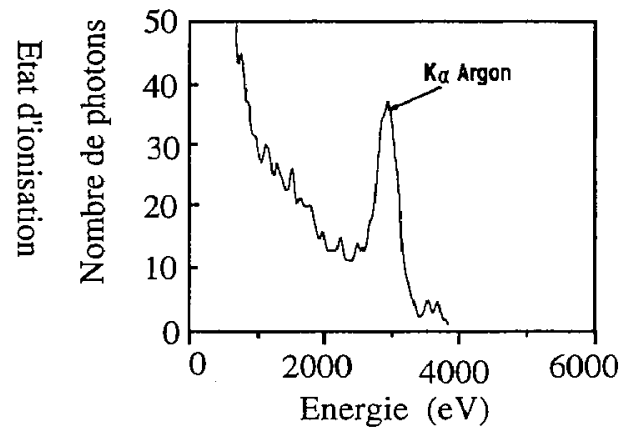

Fig 2. Emission $\mathrm{K} \alpha$ dans le jet d'Argon

L'émission $\mathrm{K} \alpha$ observée (Fig 2) apparait très sensible aux conditions de focalisation dans le jet et présente un maximum en bord de jet. Cette émission peut être interprétée comme résultant de l'ionisation des couches internes de l'Argon par des électrons d'énergie supérieure $3.2 \mathrm{KeV}$ (4). La zone d'émission à un rayon d'environ $10 \mu \mathrm{m}$ et une longueur d'environ $100 \mu \mathrm{m}$ le long de l'axe laser en bon accord avec l' étude de l'état d'ionisation moyen de l'Argon dans la zone de Rayleigh .

\section{CONCLUSION}

Cette étude montre, la possibilité de réaliser une source $X$ pulsée ultra-courte, hautement répétitive et reproductible, par irradiation par une impulsion laser femtoseconde d'un jet pulsé d'Argon haute pression. Elle constitue par ailleurs la première mise en évidence $\mathrm{d}^{\prime}$ une émission $\mathrm{K} \alpha$ associée à l' ionisation directe des couches internes de l'Argon par des électrons dont l'énergie est acquise par oscillation dans un champ laser intense.

1 P.Monot et al J. optical Soc. Am B, 9 (1992), 1579

2 S.C Rae, Optics Communication, 97 (1993), 25

3.C. Stenz et al, 24th Annual Anomalous Absorption Conference, Monterey (1994)

4.P. Audebert et al, Generation and application of ultrashort X-Ray pulses, Salamanca (1994) 